\title{
Roles of Integration of Multiple Simulation Technologies in Insights into Binding Mechanism of Inhibitors to BRD Family
}

\author{
Shiliang Wu \\ School of Science, Shandong Jiaotong University, Jinan, China
}

Email address:

wushiliang1981@163.com

\section{To cite this article:}

Shiliang Wu. Roles of Integration of Multiple Simulation Technologies in Insights into Binding Mechanism of Inhibitors to BRD Family. Advances in Bioscience and Bioengineering. Vol. 9, No. 1, 2021, pp. 13-19. doi: 10.11648/j.abb.20210901.13

Received: March 16, 2021; Accepted: March 30, 2021; Published: April 12, 2021

\begin{abstract}
Insights into binding mechanism of inhibitors to targets are expected to provide a meaningfully theoretical guidance for design and development of effective inhibitors inhibiting of the activity of targets. It is well known that the bromodomain (BRD) family has been thought as a promising target utilized for treating various human diseases, such as inflammatory disorders, malignant tumors, acute myelogenous leukemia (AML), bone diseases, etc. In this work, we summarize the roles of integration of multiple simulation technologies in exploring atomic-level dynamics changes of the BRD family because of inhibitor bindings. Molecular dynamics (MD) simulations, binding free energy calculations, calculations of dynamics cross-correlation maps (DCCMs), and principal component (PC) analysis are integrated together to uncover binding modes of inhibitors to BRDs. The results obtained from binding free energy calculations can measure binding ability of inhibitors to BRDs, and explore the main driving forces of the binding of inhibitors to BRDs. The information stemming from PC analysis can reveal the changes in conformations, internal dynamics and movement patterns of BRDs due to inhibitor associations. Residue-based free energy decomposition method is wielded to unveil contributions of separate residues to inhibitor bindings, and explore the decisive factors that affect the bindings of inhibitors to BRDs.
\end{abstract}

Keywords: Molecular Dynamics Simulations, Principal Component Analysis, MM-GBSA

\section{Introduction}

With fast development of computer technology, multiple simulation technologies, such as molecular dynamics (MD) simulations, binding free energy predictions, principal component (PC) analysis, dynamics cross-correlation maps (DCCMs) and residue-based free energy decomposition, are play increasing roles in identification of hot spots of inhibitor-target bindings and drug design. Increasing researchers are applying these simulation technologies to probe molecular mechanism with regard to inhibitor-target bindings. For example, Wang et al. explored molecular mechanisms of inhibitor bindings to bromodomain-containing protein 4 (BRD4) using MD simulations and calculations of binding free energies, and the results not only demonstrate that the associations of inhibitors obviously affect the flexibility, conformational changes, motion modes, and internal dynamics of BRD4 (1), but also show that residues Gln85,
Val87, Leu92, Leu94, Cys136 and Ile146 produce the $\mathrm{CH}-\pi$ interactions with inhibitors, while the residues Trp81, Pro82, Phe83 and Tyr139 form the $\pi-\pi$ interactions [1]. Su et al. studied the selective mechanism of inhibitors toward BRD4, BRD7 and BRD9, and the results show that Ile164 and Asn211 in BRD7 and Ile53 and Asn100 in BRD9 play a significant role in the selectivity of inhibitor H1B to BRD7 and BRD9, in addition, several key residues Phe44, Ile53, Asn100, Thr104 in BRD9 and Pro82, Lys91, Asn140, Asp144 in BRD4 also provide significant contributions to binding selectivity of inhibitors toward BRD9 and BRD4 [2]. In summary, the efficient integration of these simulation technologies will be of significance for design of potent inhibitors toward targets. Based on this aim, we design a procedure for integrating multiple technologies and it is shown in Figure 1. To shed light on this procedure, we use the BRD family that we are focusing and the AMBER 18 as an example. In the first module, the structures of BRD coupled 
with inhibitor can be obtained from the Protein Data Bank (PDB) or molecular docking. In the second module, conventional MD (cMD) and multiple replica molecular dynamics (MRMD) simulations are employed to produce structural ensembles that are used to perform statistical analyses or post-processing analyses. In the third module, post-processing analyses are carried out to probe dynamics characteristic, calculate binding free energies and decode conformational changes. In the last module, based on the information obtained from the third module, molecular mechanisms involved in inhibitor-BRD bindings are clarified. Although this work doesn't provide a novel method to study inhibitor-BRD bindings, the procedure summarized by us also provide certain guidance for the researchers who will apply simulation technologies to study inhibitor-target bindings. The details for performing integration of multiple simulation technologies are clarified as below.

\section{Materials and Methods}

\subsection{System Initialization}

The existing crystal structures of inhibitor-target complexes can be extracted from the Protein Data Bank (PDB) to obtain coordinates of MD simulations. If the crystal structures of inhibitor-BRD compounds are unavailable, the designed inhibitors can docked into the binding pocket of BRD by using the docking softwares, such as PyMOL [3] and Autodock 4.2 software [4]. The LEAP module in AMBER18 is wielded to link missing hydrogen atoms to the corresponding heavy atoms [5]. The PROPKA program is used to recognize the rational protonation states of the residues at $\mathrm{pH}=7.0$ [6]. The force field parameters for MD simulations of inhibitor stem from General Amber force field (GAFF) [7], that of protein arise from the Amber ff14SB force field [8] and that of water molecules for reflecting solvent environment originate from the TIP3P model [9]. Bond-charge correction (BCC) charges of atoms are calculated by using the AM1 method and then $\mathrm{BBC}$ charges are assigned to separate atoms of the inhibitors by making use of the ANTECHAMBE program of AMBER 18 [10]. Then the right counterions are added to neutralize the charges of each simulated systems.

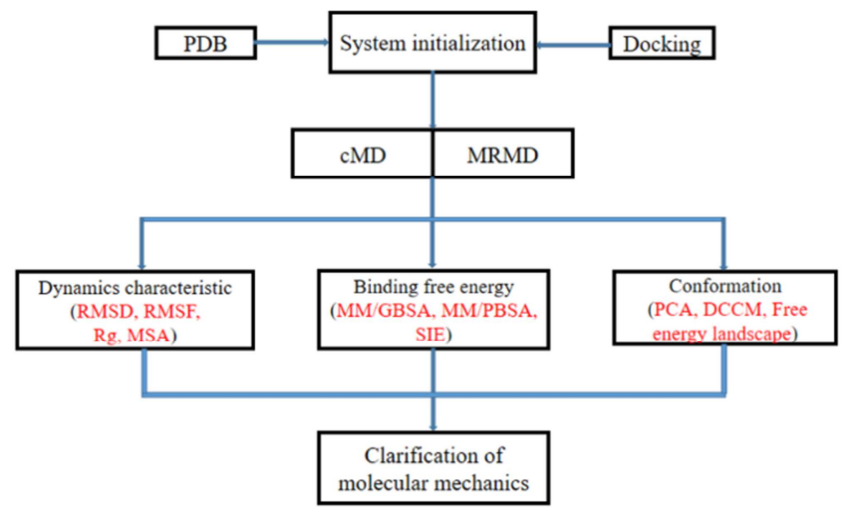

Figure 1. Scheme of multiple simulation technologies, in which multiple simulation technologies play significant roles in decoding molecular mechanism of inhibitor-target bindings.

\subsection{Molecular Dynamics Simulations}

The PMEMD module in AMBER18 is used to execute energy minimizations and MD simulations [11-13]. A two-stage energy optimization is performed on each system to eliminate close contacts and highly energetic orientations between atoms: (1) to optimize the solvent by constrain solute by a rational force constant, (2) to optimization the entire system without restriction through the steepest descent minimization of 2500 steps and the conjugate gradient minimization of 2500 steps. Then, each system is slowly heated from 0 to $300 \mathrm{~K}$ in $2 \mathrm{~ns}$ followed by the system equilibration of another $1 \mathrm{~ns}$ at $300 \mathrm{~K}$. Finally, each system is fully relaxed through 200-ns unconstrained cMD simulations at $300 \mathrm{~K}$ and $1 \mathrm{~atm}$ by using the periodic boundary condition and a time step of $2 \mathrm{fs}$. The length of simulation time can be adjusted according to different simulation systems. During simulations, a cutoff value of $10 \AA$ is used to treat van der Waals and electrostatic interactions. The SHAKE algorithm is utilized to limit the expansion and contraction of all covalent bonds connecting to hydrogen atoms [14]. The Particle Mesh Ewald (PME) method is wielded to calculate long-range electrostatic interactions [15]. The Langevin thermostat is used to adjust the temperature of each system [16].

Compared to a single long cMD simulation, MRMD simulations can be obtain better sampling efficiency. The different works verify that MRMD simulations obtain more rational results in insights into inhibitor-target binding than a single long cMD simulation [17-21].

\section{Results}

\subsection{Dynamics Characteristic}

The CPPTRAJ module in AMBER18 is used to calculate root mean square deviation (RMSD) which can be utilized to evaluate the stability of MD simulations (Figure 2A), while the root mean square fluctuation (RMSF) of the $C_{\alpha}$ atoms in BRDs computed by utilizing the equilibrated parts of $\mathrm{MD}$ trajectory is used to explore effect of inhibitor bindings on the structural flexibility of BRDs (Figure 2B). In addition, molecular surface areas (MSAs) are used to probe the total expansion or contraction of BRDs (Figure 3), while the radius of gyrations (RGs) are also employed to intuitively observe the ductility of BRDs in spatial distribution (Figure 4).

\subsection{The Conformational Changes of Protein}

The dynamics cross-correlation map (DCCM) is an effective method to explore internal dynamics of targets [22-26]. The cross-correlation coefficient $C(i, j)$ is determined by the following equation (1)

$$
C(i, j)=\frac{\left.<\Delta r_{i} \cdot \Delta r_{j}\right\rangle}{\left(<\Delta r_{i}^{2}><\Delta r_{j}^{2}>\right)^{1 / 2}}
$$

where $\langle\cdots\rangle$ indicates an ensemble average over the structures recorded in the MD trajectory, $\Delta r_{i}$ represents the 

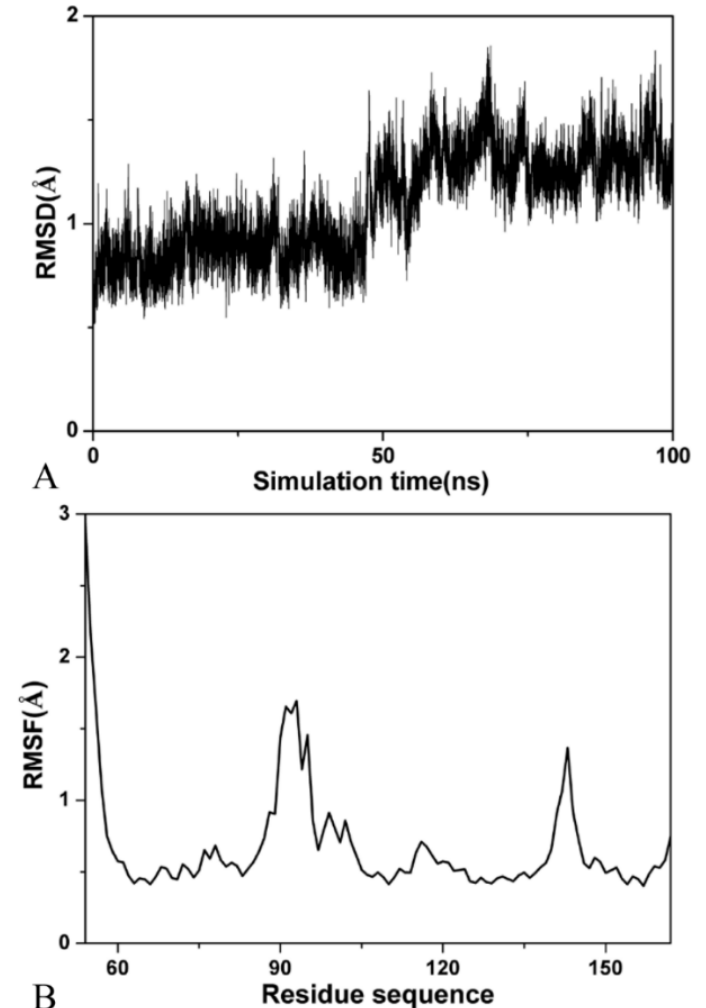

Figure 2. Structural fluctuation and structural flexibility: (A) Root-mean-square deviation (RMSD) of backbone atoms in BRD relative to their initial structures as function of simulations time, (B) Root-mean-square fluctuation (RMSF) of $C_{\alpha}$ atoms in BRD of the simulated system.

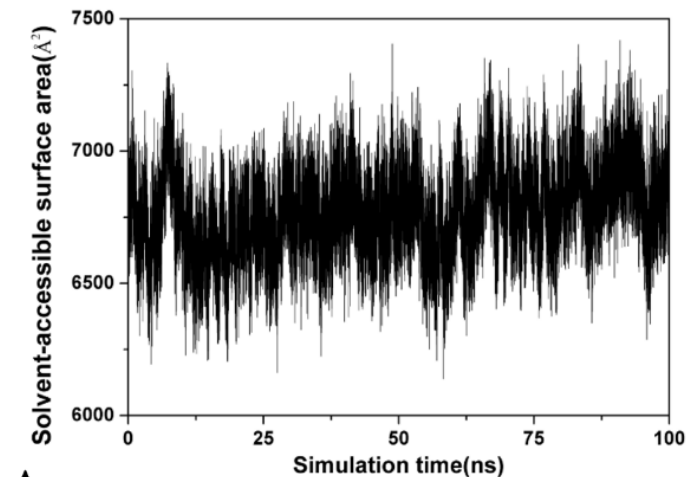

A

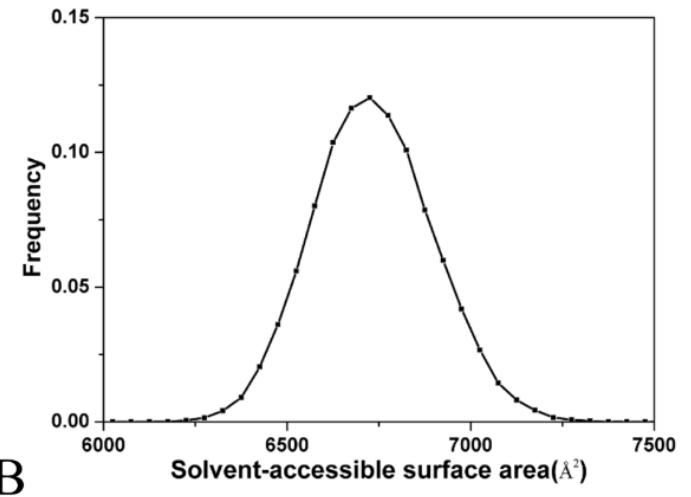

Figure 3. (A) Molecular surface area (MSA) as function of simulations time, (B) Frequency distributions of MSA, in which the $x$-axis shows the MSA and the y-axis indicates the frequency distribution of the MSA.
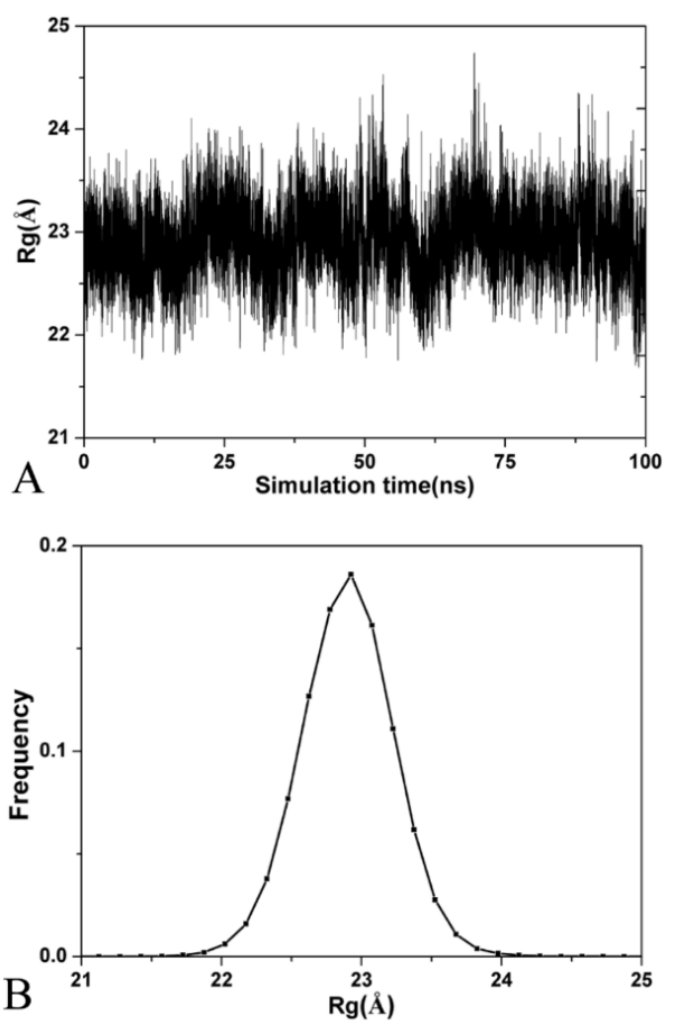

Figure 4. (A) Radius of gyration (Rg) as function of simulations time, (B) Frequency distribution of $R g$, in which the $x$-axis indicates the radius of gyrations and the y-axis represents frequency distribution.

deviation between the instantaneous position of the $C_{\alpha}$ atom in the $i$ th residue and its average position. The value range of $C(i, j)$ is located between -1 to +1 , from which $C(i, j)>0$ indicates a positively correlated motions, while $C(i, j)<0$ indicates an anti-correlated motions. The CPPTRAJ module in AMBER 18 is used to calculate $C(i, j)$ [27]. The color-coded style is usually utilized to reflect the extents of correlated movements, in which the red and yellow show strongly positive correlated movements (the regions R1 and R3 in Figure 5A), while the blue and dark blue represent strongly anti-correlated movements (the regions R2 and R4 in Figure $5 \mathrm{~A})$.

Principal components analysis (PCA) can be used to explore the conformational alterations of functional significance domains in targets $[28,29]$. To perform the PCA, firstly a covariance matrix $C$ is constructed by using the coordinates of the $\mathrm{C}_{\alpha}$ atoms recorded at the MD trajectory according to the equation (2) as below

$$
C=<\left(r_{i}-<r_{i}>\right)\left(r_{j}-<r_{j}>\right)^{T}>(i, j=1,2,3, \cdots, 3 N)
$$

where $r_{i}$ indicates the Cartesian coordinates of the $C_{\alpha}$ atom in the $i$ th residue and the symbol $N$ represents the number of the $C_{\alpha}$ atoms of BRD. The BRD structure extracted from MD trajectory is superimposed on the reference structure to eliminate all translations and rotations. Then the average is calculated over the structures recorded in the MD trajectory. The covariance matrix $C$ in equation (2) is symmetric and can be transformed into a diagonal matrix $\Lambda$ of eigenvalues $\lambda_{i}$ through an orthogonal transformation matrix $T$ : 

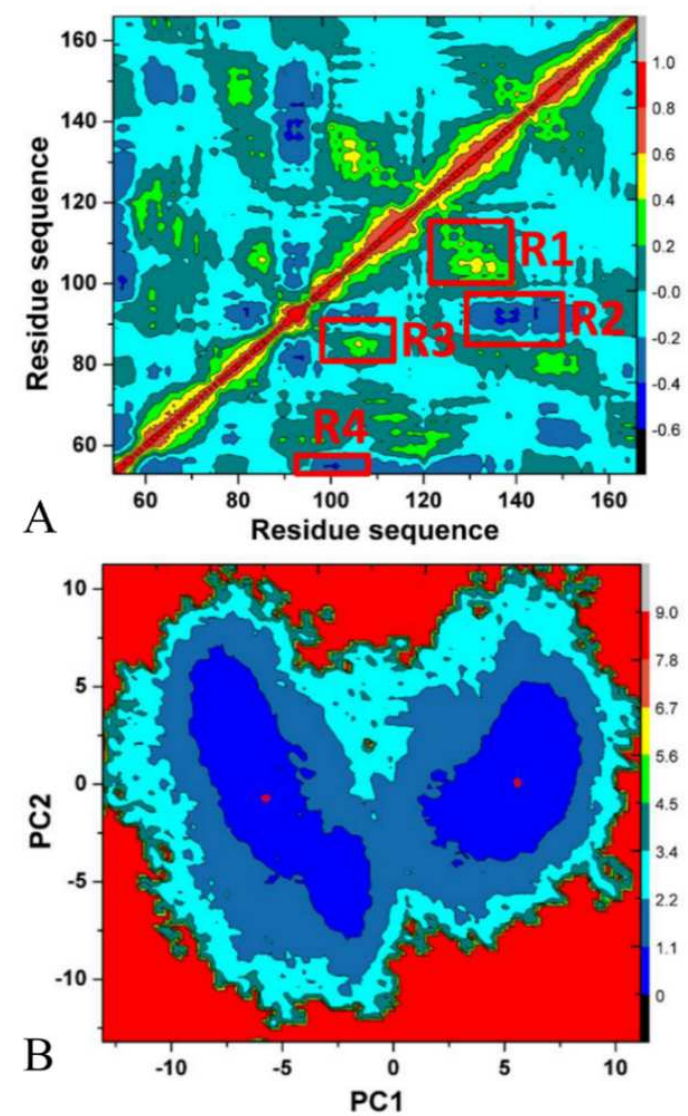

Figure 5. Correlated motions and free energy landscapes: (A) Dynamics cross-correlation map (DCCM) of the $C_{\alpha}$ atoms in BRD over their average positions based on the equilibrium trajectory of $M D$ simulation, (B) free-energy landscapes built by employing projections of MD trajectory on the first two principal components $P C 1$ and $P C 2$ arising from the diagonalization of covariance matrix.

$$
\Lambda=T^{T} C_{i, j} T
$$

where the columns are the eigenvectors correlating with eigenvalues and the eigenvectors corresponding to the first few bigger eigenvalues can better characterize the concerted motions of the domains in BRD. Figure 6 shows the relationship between the eigenvalues and the eigenvector index. Generally, the larger eigenvalues correspond to the significantly concerted movements of the domains in BRD, while the smaller eigenvalues describe more constrained and local fluctuations.

Free-energy landscapes that are constructed by wielding the projections of the MD trajectory on the first two eigenvectors as the reaction coordinates can be employed to further explore the conformational changes. As indicated in Figure 5B, the BRDs span two conformational spaces and fall into two energy basins, implying that the conformations of an exampled BRD exist in two energy states.

\subsection{Binding Free Energy and Binding Mechanism}

Molecular mechanics/Poisson-Boltzmann surface area (MM-PBSA) and MM-GBSA have been widely used to estimate binding free energies [30-34]. Hou et al. reported that MM-GBSA method exhibits better performance at the rank of

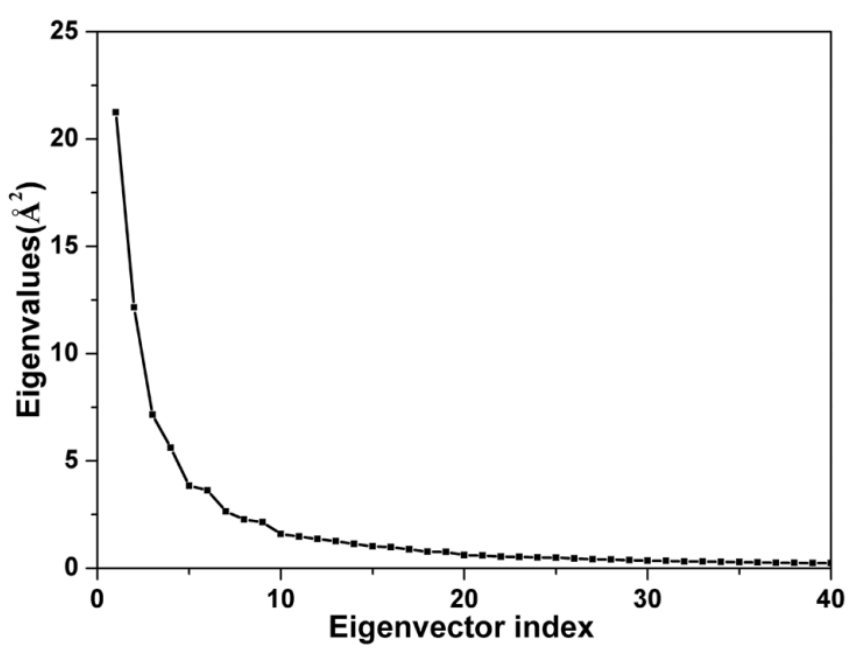

Figure 6. The eigenvalues plotted against the corresponding eigenvector indices obtaining from the $C_{\alpha}$ covariance matrix constructed by using the MD trajectory.

the binding abilities for the systems without metals than MM-PBSA method, and their comparisons provide a hint for selecting MM-PBSA or MM-GBSA as the efficient tool to predict binding free energies of inhibitors to targets $[35,36]$. Usually, a number of snapshots taken from the equilibrated part of MD trajectory are utilized to perform binding free energy calculations. In MM-PBSA and MM-GBSA, the binding free energies include five terms: electrostatic $\left(\Delta E_{\text {ele }}\right)$ interactions and van der Waals $\left(\Delta E_{v d w}\right)$ interactions in the gas phase, non-polar $\left(\Delta G_{\text {nonpol }}\right)$ contributions and polar $\left(\Delta G_{p o l}\right)$ contributions to solvation free energies, and the entropy contribution to inhibitor bindings $(-T \Delta S)$. The equation can be written as the following:

$$
\Delta G=\Delta E_{v d w}+\Delta E_{\text {ele }}+\Delta G_{\text {nonpol }}+\Delta G_{\text {pol }}-T \Delta S
$$

in which $\Delta E_{v d w}$ and $\Delta E_{\text {ele }}$ are calculated by employing ff14SB force field and molecular mechanics. The third term $\Delta G_{\text {nonpol }}$ can be estimated from the equation as follow:

$$
\Delta G_{\text {nonpol }}=\gamma \times(\mathrm{SASA})+\beta
$$

where SASA is the solvent-accessible surface area, the empirical constants $\gamma=0.0072 \mathrm{kcal} / \mathrm{mol} \cdot \AA^{-2}$, while $\beta=0.0 \mathrm{kcal} / \mathrm{mol}$. The fourth term $\Delta G_{p o l}$ can be calculated by using the MM-GBSA module in AMBER18. The last term $-T \Delta S$ is generally computed as the sum of translational, rotational, and vibrational entropies by means of classical thermodynamics and normal mode analysis. Due to computationally expensive calculations of the entropy, only a small number of conformations taken from the aforementioned snapshots performing binding free energy calculations were adopted for estimation of the entropy contributions. In addition, to explore the contribution of per residue to the binding free energy, the residue-based free energy decomposition method is also used to calculate interactions of inhibitor with each residue in BRDs.

SIE method also can provide fast and rational calculation of binding free energies [37, 38]. In SIE method, binding free energies of inhibitors to targets are calculated as following: 


$$
\Delta G_{\text {bind }}=\alpha \times\left[\Delta E_{v d W}+\Delta E_{c}\left(D_{\text {in }}\right)+\Delta G^{R}+\gamma \Delta M S A(\rho)\right]+C
$$

where $\Delta E_{v d W}$ and $\Delta E_{c}$ represent van der Waals interactions and intermolecular Coulomb interactions in the bound state, respectively. $\Delta G^{R}$ is the change in the reaction field energy induced by inhibitor binding. The forth term $\gamma \Delta M S A(\rho)$ is related to the change in the molecular surface area upon binding. The parameter $\alpha$ corresponds to the global proportionality coefficient related to the loss of conformational entropy upon binding. The parameters $D_{i n}, \gamma, \rho$ and $C$ indicate the solute interior dielectric constant, the molecular surface area coefficient, Amber van der Waals radii linear scaling coefficient, and a constant, respectively. The values of five parameters are $\alpha=0.1048, D_{\text {in }}=2.25, \gamma=0.0129 \mathrm{kcal} /\left(\mathrm{mol} \cdot \AA^{2}\right)$, $\rho=1.1$ and $C=-2.89 \mathrm{kcal} / \mathrm{mol}$, respectively.

The SIE calculations are performed with the program Sietraj. Table 1 shows binding free energies of inhibitors to BRD computed with MM-GBSA method and SIE methods, separately.

Table 1. Binding free energies of inhibitor to BRD computed with MM-GBSA method and SIE method ${ }^{a}$.

\begin{tabular}{llll}
\hline Terms & MM-GBSA method & Terms & SIE method \\
\hline$\Delta E_{\text {ele }}$ & $-28.05 \pm 0.49$ & $\Delta E_{c}$ & $-11.95 \pm 0.39$ \\
$\Delta E_{\text {vdw }}$ & $-46.37 \pm 0.21$ & $\Delta E_{v d w}$ & $-45.64 \pm 0.41$ \\
$\Delta G_{\text {pol }}$ & $44.16 \pm 0.46$ & $\Delta G^{R}$ & $16.39 \pm 0.37$ \\
$\Delta G_{\text {nopol }}$ & $-4.17 \pm 0.02$ & $\gamma \Delta M S A$ & $-8.50 \pm 0.05$ \\
$-T \Delta S$ & $22.02 \pm 0.38$ & ${ }^{\mathrm{b}}--$ & - \\
${ }^{\mathrm{c}} \Delta G_{\text {bind }}$ & -12.41 & ${ }^{\mathrm{d}} \Delta G_{\text {bind }}$ & -8.10 \\
${ }^{\mathrm{e}} \Delta G_{\text {exp }}$ & -9.37 & $\Delta G_{\text {exp }}$ & -9.37 \\
\hline
\end{tabular}

${ }^{\mathrm{a}}$ All values are in $\mathrm{kcal} / \mathrm{mol}$.

${ }^{\mathrm{b}}$ The data is not available.

${ }^{\mathrm{c}} \Delta G_{\text {bind }}=\Delta E_{\text {ele }}+\Delta E_{\text {vdw }}+\Delta G_{\text {pol }}+\Delta G_{\text {nopol }}-T \Delta S$.

${ }^{\mathrm{d}} \Delta G_{\text {bind }}=\alpha \times\left[\Delta E_{c}\left(D_{\text {in }}\right)+\Delta E_{v d W}+\Delta G^{R}+\gamma \Delta M S A(\rho)\right]+C$

${ }^{\mathrm{e}}$ The experimental values were obtained from the experimental IC50 values in reference [39] by employing the equation $\Delta G_{\text {exp }} \approx R T \operatorname{lnIC} 50$.

\subsection{Decomposition of Binding Affinity into Contributions of Individual Residues}

Analysis of residue-based free energy decomposition can elucidate the role of a single residue in inhibitor associations. To gain a further insight into binding of pivotal residues with the inhibitors, the residue-based free energy decomposition method is applied to compute the interactions of inhibitors with separate residues in BRDs using the snapshots same as the calculations of binding free energies (Figure 7A). On the basis of Figure $7 \mathrm{~A}$, the interactions of four residues with inhibitor are stronger than $1 \mathrm{kcal} / \mathrm{mol}$, and these residues are Trp81, Val87, Leu92, and Ile146. The geometric positions of important residues relative to inhibitors are displayed by using the lowest energy structures extracted from the MD simulations (Figure 7B). Figure 7B shows that the residues of BRDs mainly produce hydrophobic interactions with inhibitors, such as $\mathrm{CH}-\pi$ and $\pi-\pi$ interactions. The interactions of residues with the inhibitors rationally explains internal dynamics and conformational changes of BRDs due to inhibitor bindings.
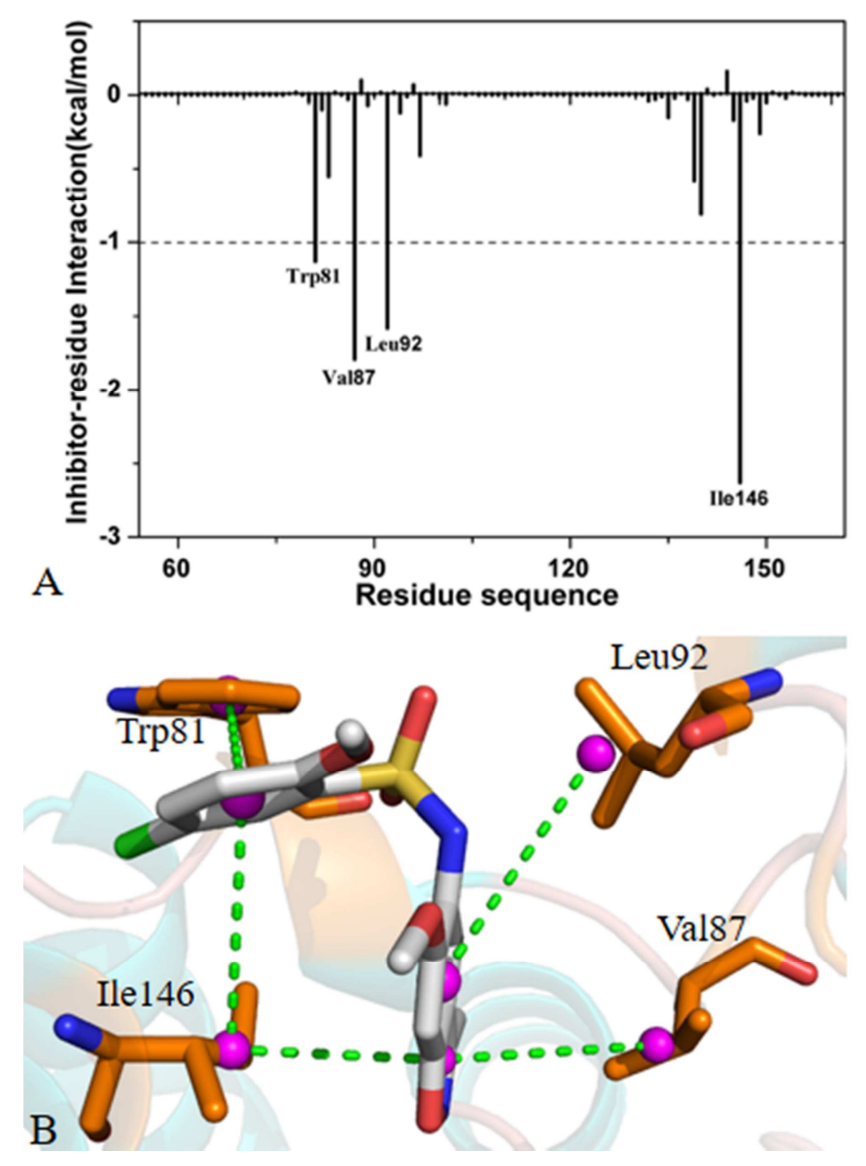

Figure 7. Inhibitor-residue interaction spectrum: (A) Interactions of inhibitor with separate residues in BRD, (B) Geometric positions of inhibitor relative to key residues of $B R D$ involving strong interaction.

\section{Conclusion}

In this work, multiple simulation technologies, such as molecular dynamics (MD) simulations, binding free energy calculations, calculations of dynamics cross-correlation maps (DCCMs), and principal component (PC) analysis are integrated together to explore atomic-level dynamics changes of the bromodomain (BRD) family because of inhibitor bindings. The results obtained from binding free energy calculations can measure binding ability of inhibitors to BRDs. The information coming from PC analysis can reveal the changes in internal dynamics and movement patterns of BRDs due to the bindings of inhibitor. Residue-based free energy decomposition method is wielded to unveil contributions of separate residues to inhibitor bindings. Although this work does not provide a novel method to study inhibitor-BRD bindings, the procedure summarized by us also provide useful helps and certain guidance for the researchers who will apply simulation technologies to study inhibitor-target bindings.

\section{Acknowledgements}

This work is supported by Science Foundation of Shandong Jiaotong University (No. Z201206). 


\section{References}

[1] L. Wang, Y. Wang, H. Sun, J. Zhao, Q. Wang, Theoretical insight into molecular mechanisms of inhibitor bindings to bromodomain-containing protein 4 using molecular dynamics simulations and calculations of binding free energies, Chem. Phys. Lett. 736 (2019) 136785.

[2] J. Su, X. Liu, S. Zhang, F. Yan, Q. Zhang, J. Chen, Insight into selective mechanism of class of I-BRD9 inhibitors toward BRD9 based on molecular dynamics simulations, Chem. Biol. Drug. Des. 93 (2019) 163-176.

[3] W. L. DeLano, The PyMOL molecular graphics system, Available at http://www.pymol.org.

[4] D. S. Goodsell, G. M. Morris, A. J. Olson, Automated docking of flexible ligands: applications of AutoDock, J. Mol. Recognit. 9 (1996) $1-5$.

[5] D. A. Case, T. E. Cheatham, 3rd, T. Darden, H. Gohlke, R. Luo, K. M. Merz, Jr., A. Onufriev, C. Simmerling, B. Wang, R. J. Woods, The Amber biomolecular simulation programs, J. Comput. Chem. 26 (2005) 1668-1688.

[6] H. Li, A. D. Robertson, J. H. Jensen, Very fast empirical prediction and rationalization of protein $\mathrm{pKa}$ values, Proteins 61 (2005) 704-721.

[7] J. Wang, R. M. Wolf, J. W. Caldwell, P. A. Kollman, D. A. Case, Development and testing of a general amber force field, J. Comput. Chem. 25 (2004) 1157-1174.

[8] J. A. Maier, C. Martinez, K. Kasavajhala, L. Wickstrom, K. E. Hauser, C. Simmerling, ff14SB: Improving the Accuracy of Protein Side Chain and Backbone Parameters from ff99SB, J. Chem. Theory Comput. 11 (2015) 3696-3713.

[9] W. L. Jorgensen, J. Chandrasekhar, J. D. Madura, R. W. Impey, M. L. Klein, Comparison of simple potential functions for simulating liquid water, J. Chem. Phys. 79 (1983) 926-935.

[10] A. Jakalian, D. B. Jack, C. I. Bayly, Fast, efficient generation of high-quality atomic charges. AM1-BCC model: II. Parameterization and validation, J. Comput. Chem. 23 (2002) 1623-1641.

[11] R. Salomon-Ferrer, A. W. Götz, D. Poole, S. Le Grand, R. C. Walker, Routine Microsecond Molecular Dynamics Simulations with AMBER on GPUs. 2. Explicit Solvent Particle Mesh Ewald, J. Chem. Theory Comput. 9 (2013) 3878-3888.

[12] J. Chen, X. Wang, T. Zhu, Q. Zhang, J. Z. H. Zhang, A Comparative Insight into Amprenavir Resistance of Mutations V32I, G48V, I50V, I54V, and I84V in HIV-1 Protease Based on Thermodynamic Integration and MM-PBSA Methods, J. Chem. Inf. Model. 55 (2015) 1903-1913.

[13] J. Chen, X. Wang, L. Pang, J. Z. H. Zhang, T. Zhu, Effect of mutations on binding of ligands to guanine riboswitch probed by free energy perturbation and molecular dynamics simulations, Nucleic Acids Res. 47 (2019) 6618-6631. doi: 10.1093/nar/gkz499.

[14] T. G. Coleman, H. C. Mesick, R. L. Darby, Numerical integration: a method for improving solution stability in models of the circulation, Ann Biomed. Eng. 5 (1977) 322-328.
[15] U. Essmann, L. Perera, M. L. Berkowitz, T. Darden, H. Lee, L. G. Pedersen, A smooth particle mesh Ewald method, J. Chem. Phys. 103 (1995) 8577-8593.

[16] J. A. Izaguirre, D. P. Catarello, J. M. Wozniak, R. D. Skeel, Langevin stabilization of molecular dynamics, J. Chem. Phys. 114 (2001) 2090-2098.

[17] J. Chen, X. Liu, S. Zhang, J. Chen, H. Sun, L. Zhang, Q. Zhang, Molecular mechanism with regard to the binding selectivity of inhibitors toward FABP5 and FABP7 explored by multiple short molecular dynamics simulations and free energy analyses, Phys. Chem. Chem. Phys. 22 (2020) 2262-2275.

[18] P. Auffinger, E. Westhof, RNA hydration: three nanoseconds of multiple molecular dynamics simulations of the solvated tRNA (Asp) anticodon hairpin, J. Mol. Biol. 269 (1997) 326-341.

[19] L. S. Caves, J. D. Evanseck, M. Karplus, Locally accessible conformations of proteins: multiple molecular dynamics simulations of crambin, Protein Sci. 7 (1998) 649-666.

[20] J. Chen, J. Wang, B. Yin, L. Pang, W. Wang, W. Zhu, Molecular Mechanism of Binding Selectivity of Inhibitors toward BACE1 and BACE2 Revealed by Multiple Short Molecular Dynamics Simulations and Free-Energy Predictions, ACS Chem. Neurosci. 10 (2019) 4303-4318.

[21] S. Tian, J. Zeng, X. Liu, J. Chen, J. Z. H. Zhang, T. Zhu, Understanding the selectivity of inhibitors toward PI4KIII and PI4KIII $\beta$ based molecular modeling, Phys. Chem. Chem. Phys. 21 (2019) 22103-22112.

[22] T. Ichiye, M. Karplus, Collective motions in proteins: a covariance analysis of atomic fluctuations in molecular dynamics and normal mode simulations, Proteins 11 (1991) 205-217.

[23] J. Chen, W. Wang, L. Pang, W. Zhu, Unveiling conformational dynamics changes of H-Ras induced by mutations based on accelerated molecular dynamics, Phys. Chem. Chem. Phys. 22 (2020) 21238-21250.

[24] R. M. Levy, A. R. Srinivasan, W. K. Olson, J. A. McCammon, Quasi-harmonic method for studying very low frequency modes in proteins, Biopolymers 23 (1984) 1099-1112.

[25] J. Chen, B. Yin, W. Wang, H. Sun, Effects of Disulfide Bonds on Binding of Inhibitors to $\beta$-Amyloid Cleaving Enzyme 1 Decoded by Multiple Replica Accelerated Molecular Dynamics Simulations, ACS Chem. Neurosci. 11 (2020) 1811-1826.

[26] J. Chen, J. Wang, F. Lai, W. Wang, L. Pang, W. Zhu, Dynamics revelation of conformational changes and binding modes of heat shock protein 90 induced by inhibitor associations, RSC Adv. 8 (2018) 25456-25467.

[27] D. R. Roe, T. E. Cheatham, 3rd, PTRAJ and CPPTRAJ: Software for Processing and Analysis of Molecular Dynamics Trajectory Data, J. Chem. Theory Comput. 9 (2013) 3084-3095.

[28] A. D. McLachlan, Gene duplications in the structural evolution of chymotrypsin, J. Mol. Biol. 128 (1979) 49-79.

[29] A. Amadei, A. B. M. Linssen, H. J. C. Berendsen, Essential dynamics of proteins, Proteins: Structure, Function, and Bioinformatics 17 (1993) 412-425. 
[30] W. Wang, P. A. Kollman, Computational study of protein specificity: the molecular basis of HIV-1 protease drug resistance, Proc. Natl. Acad. Sci. U. S. A. 98 (2001) 14937-14942.

[31] J. Wang, P. Morin, W. Wang, P. A. Kollman, Use of MM-PBSA in reproducing the binding free energies to HIV-1 RT of TIBO derivatives and predicting the binding mode to HIV-1 RT of efavirenz by docking and MM-PBSA, J. Am. Chem. Soc. 123 (2001) 5221-5230.

[32] J. Chen, W. Wang, H. Sun, L. Pang, B. Yin, Mutation-mediated influences on binding of anaplastic lymphoma kinase to crizotinib decoded by multiple replica Gaussian accelerated molecular dynamics, J. Comput. Aid. Mol. Des. 34 (2020) 1289-1305.

[33] S. L. Wu, L. F. Wang, H. B. Sun, W. Wang, Y. X. Yu, Probing molecular mechanism of inhibitor bindings to bromodomain-containing protein 4 based on molecular dynamics simulations and principal component analysis, SAR QSAR Environ. Res. 31 (2020) 547-570.

[34] F. Yan, X. Liu, S. Zhang, J. Su, Q. Zhang, J. Chen, Effect of double mutations $\mathrm{T} 790 \mathrm{M} / \mathrm{L} 858 \mathrm{R}$ on conformation and drug-resistant mechanism of epidermal growth factor receptor explored by molecular dynamics simulations, RSC Adv. 8 (2018) 39797-39810.
[35] T. Hou, J. Wang, Y. Li, W. Wang, Assessing the performance of the MM/PBSA and MM/GBSA methods. 1. The accuracy of binding free energy calculations based on molecular dynamics simulations, J. Chem. Inf. Model. 51 (2011) 69-82.

[36] H. Sun, Y. Li, S. Tian, L. Xu, T. Hou, Assessing the performance of MM/PBSA and MM/GBSA methods. 4. Accuracies of MM/PBSA and MM/GBSA methodologies evaluated by various simulation protocols using PDBbind data set, Phys. Chem. Chem. Phys. 16 (2014) 16719-16729.

[37] M. Naïm, S. Bhat, K. N. Rankin, S. Dennis, S. F. Chowdhury, I. Siddiqi, P. Drabik, T. Sulea, C. I. Bayly, A. Jakalian, E. O. Purisima, Solvated interaction energy (SIE) for scoring protein-ligand binding affinities. 1. Exploring the parameter space, J. Chem. Inf. Model. 47 (2007) 122-133.

[38] J. Chen, J. Wang, W. Zhu, Binding modes of three inhibitors 8CA, F8A and I4A to A-FABP studied based on molecular dynamics simulation, PLoS One 9 (2014) e99862.

[39] J. Zhu, J. Dong, L. Batiste, A. Unzue, A. Dolbois, V. Pascanu, P. Śledź, C. Nevado, A. Caflisch, Binding Motifs in the CBP Bromodomain: An Analysis of 20 Crystal Structures of Complexes with Small Molecules, ACS Med. Chem. Lett. 9 (2018) 929-934. 